\title{
Tracing and Enhancing Serendipitous Learning with ViewpointS
}

\author{
Stefano A. Cerri ${ }^{1}$ and Philippe Lemoisson ${ }^{2}$ \\ ${ }^{1}$ LIRMM: University of Montpellier and CNRS, France \\ cerri@lirmm.fr \\ ${ }^{2}$ UMR Territories, Environment, Remote Sensing and Spatial Information, CIRAD, Montpel- \\ lier, France \\ philippe.lemoissonecirad.fr
}

\begin{abstract}
This is a position paper describing the author's views on a potential new research direction for assessing, constructing and exploiting brainfounded models of learning of individual as well as collective humans. The recent approach - called ViewpointS - aiming to unify the Semantic and the Social Web, data mining included, by means of a simple "subjective" primitive the viewpoint - denoting proximity among elements of the world, seems to offer a promising context of innovative empirical research in modeling human learning less constrained with respect to the previous three other ones. Within this context, a few phenomena of serendipitous learning have been simulated, showing that the process of collective construction of knowledge during free navigation may offer interesting side effects of informal, serendipitous knowledge acquisition and learning. We envision therefore an extension of the modeling functions within ViewpointS by adding measures of the emotions and mental states as acquired during experimental sessions. These brain-related components may in a first phase allow to describe and classify models in order to understand the relations among knowledge structures and mental states. Subsequently, more predictive experiments may be envisaged. These may allow to forecast the acquisition of knowledge as well as sentiment from previous events during interactions. We are convinced that useful applications may range, for instance, from Tutoring, to Health, to consensus formation in Politics at very low investment costs as the experimental set up consists of minimal extensions of the Web.
\end{abstract}

Keywords: Brain-aware individual and collective models of human learning, Serendipitous knowledge and sentiment acquisition, Informal Learning, Web-interactive Knowledge construction and exploitation, Unifying Semantic and Social Web.

\section{$1 \quad$ Introduction}

This position paper aims to contribute to the BFAL17 Conference by means of a description of potential research projects combining the state of the art of the disciplines reported in the Call. We adopt the BFAL17 view that the emergence of new assessment devices allows to foresee totally new experiments on various aspects of 
human learning, enabling a better understanding of human brain's structure and behavior about the crucial function of learning.

In particular we will capitalize from the long experience in Intelligent Tutoring that paved the way to better interactive systems enhancing learning by starting from the construction of models or profiles of community members [1], [2]. Our experience has shown that modeling is crucial in order to understand the interacting human and therefore to personalize interactions. However, modeling is extremely difficult if not intractable [3]. We aim to make model acquisition easier, as well as try to widen this modeling practice to generic interactive systems of free navigation and deepen it by adding a brain-aware component.

These experiments, however, have yet to be conceived, financed, conducted and evaluated requiring in addition the contributions of experts in different domains, each one unable to encompass the whole challenge of the enterprise.

In the following we will present our position concerning:

1. human learning "as a side effect of interactions" (informal learning [4], in particular serendipitous learning);

2. the potential exploitation of a new, emergent mode of tracing and exploiting agent-to-world (including other agents) interactions, called ViewpointS [5,6];

3. the assessment of serendipitous, informal learning effects within ViewpointS - based on interactive processes by means of neuro-physiological experiments such as those performed mainly within formal learning contexts -; and finally

4. the envisaged potential applications to learning-scientific discovery, acquiring a political conviction and health-radicalization.

\section{$2 \quad$ Serendipitous learning}

The concept of serendipitous learning is usually denoting the result of an event of learning occurring in the absence of explicit will of the learner; however it does not explain how this event occurs. We report from [7] quoting [8] that: "Like all intuitive operating, pure serendipity is not amenable to generation by a computer. The very moment I can plan or program 'serendipity' it cannot be called serendipity anymore." We may adopt most if not all the conditions for serendipity reported, yet the phenomenon is hardly to be foreseen, like other human phenomena linked to creativity. By consequence, we limit our ambition to the analysis of processes enhancing events of serendipitous learning occurring possibly during free navigation on the Web as a consequence of the availability of new knowledge structures tuned to the user.

We assume that Information access and acquisition enhances learning as a function of the learner's interest and commitment. As a consequence, we claim that

a. the proximity of Information relevant to the learner may facilitate this kind of unexpected learning during free navigation; 
b. the learner's motivation, commitment, emotions, shortly: mental state ${ }^{1}$ may facilitate this kind of learning.

Usually, we consider "free navigation" to be a process of searching among resources that are available at one click distance in a document or either in a list resulting from a search triggered by a query expressed in words. The proximity of a link to follow is then structural: a link is near if it is positioned next to my gaze when I look at the page. This position is determined by structural, logical, objective reasons.

There are other types of free navigation, however. For instance, we may freely navigate on a map from Google Maps: in this case proximity is topological, not structural: a city or a museum is near if its position is near to my gaze that looks at a point topologically near to the city or museum; latitude and longitude being the rationale for distance, i.e.: the topology is metric.

There exist also dedicated maps: they may enhance the appearance of selected objects or events. For instance, historical maps may show territories or monuments or battlefields by colors or symbols that facilitate discovering, understanding and mastering the history of the map area. These maps include knowledge that is subjective with respect to the producer.

Maps may also show proximities even without a real corresponding metric topology, as it is the case of maps of transport networks: the Information coded in the map is not strictly related to the fact that a metro station is more or less distant from the next one, rather that it is simply "the next station". Topology may thus be non metric but conceptual; tuned to the passenger's interest: what is my next station?

Another example that we all know are maps of the US - originally from Saul Steinberg - that show - in perspective - very small entities on the horizon corresponding to Europe and cities such as London or Paris or Pisa; the association of Pisa being purely due to the fact that, even if much smaller and less important than Paris or London, it is famous for its tower as a symbol of Italy or even Europe. For these maps what counts are not only the consumer's interest, but also his/her knowledge. This kind of "geographic" representation is topological, non metric and adapted to the subjective user's interests and knowledge.

We will show how to construct semi-automatically models during the construction and exploitation of an Information "geographical" map - called Knowledge Map, based on subjective proximity - that may fit the learner's cognitive state thus may be relevant to the learner and adapted to his/her mental state. As a consequence, we conjecture that this navigation may enhance serendipitous learning, discovery and acquisition.

For simplicity (and limited competence): in this paper we are not making a clear distinction among these time-dependent human properties: the word "mental state" will represent non rational aspects such as emotions, motivation, commitment, sentiment. 


\section{The interest of subjective proximity in human cognition}

In the theory and practice of the last 60 year's psychology, Rorschach tests $[9,10]$, Personality Traits tests [11,12] and Principles of grouping or Gestalt laws of grouping [13] are all based on subjective evaluations, not objective classifications. In these psychological approaches to human cognition, the subjective assessment of "proximity" among elements seems to play an important role for estimating the human mental state: for instance a certain Rorschach picture "evokes" some concepts/documents/people.

Also in the foundational work of Edelman in Physiology [14] « image evokes concept » may be interpreted as « there are strong connections between neural area 1 (image reception) and neural area 2 (abstraction $\mathrm{X}$ )» therefore « close to each other» if we choose the appropriate distance.

Vygotsky's ZPD (Zone of Proximal Development) and his vision [15], similar to the one of Piaget, may be considered another witness of the importance of "proximity" in learning processes both in spontaneous development and at school.

This does not mean that we adopt any of the above quoted visions, just that we notice that many qualified psychologists and physiologists consider subjective proximity among human cognitive structures as an important element to exploit in order to understand human cognition. Therefore, we have committed ourselves to found our investigations on human informal learning processes, in particular serendipitous learning, by means of representing and using subjective proximities rather than $o b$ jective fits.

It will be one of the subjects of our investigation to outline the correspondence of these human cognitive structures - the ones linked to proximities - with the world elements (Agents, Documents and Concepts) that we consider essentially distinguished for representation and reasoning, as outlined in the following section.

\section{Proximity in ViewpointS: Agents, Documents and Concepts}

ViewpointS is a conceptual framework, now implemented through a Web application ${ }^{2}$, enabling to represent, construct and exploit collective knowledge and intelligence in a rather innovative fashion [5]. In ViewpointS, the authors attempt to integrate the Semantic and the Social Web, data mining included, at the same time preserving subjectivity both during the construction and the exploitation of the representation.

The essential component of the model and the platform are viewpoints, i.e.: triplets declaring the proximity of an element to any other element of the reduced world. Thus ViewpointS - the model and the platform - consists of many viewpoints as well as the

\footnotetext{
${ }^{2}$ http://viewpoints.cirad.fr/ViewpointsWebApp (accessed on July $21^{\text {st }}, 2017$ )
} 
processes necessary for constructing and exploiting ${ }^{3}$ them embedded in the Web application.

By adopting this model, we assume the world to be reduced to 3 sets of elements, also called knowledge resources or resources:

1. Agents (Human and Artificial): these are active elements in the sense that they are the only ones actively declaring the proximity of any two elements. However, they are also passive in the sense that an Agent (the active one) may declare the proximity of another Agent (the passive one) with an Agent, a Document or a Classifier. Since each Agent has the right to "declare viewpoints" the declarations produced are autonomous and subjective. This is the world representing Human and - as much as metaphorically possible - Artificial Intelligence. Agents declare and exploit knowledge exhibiting therefore intelligence ${ }^{4}$. Provenance is always respected and may be used in search;

2. Documents - written, pictures, graphs, movies, real as well as virtual i.e.: numeric documents - are what the Web exhibits about the real world external to Agents and

3. Classifiers, also called Concepts - classes, subjects of a discipline, names of emotions, mental states, descriptors, names of communities, religions -. This is the world constructed by culture, language, history, science.

Proximity may be declared between two among the three sets of elements; therefore: $3 \times 2=6$ types of (undirected) proximity may be declared: Agent-Agent, AgentDocument, Agent-Concept, Document-Document, Document-Concept, ConceptConcept.

The formalization of these heterogeneous semantics is illustrated in Fig. 1:

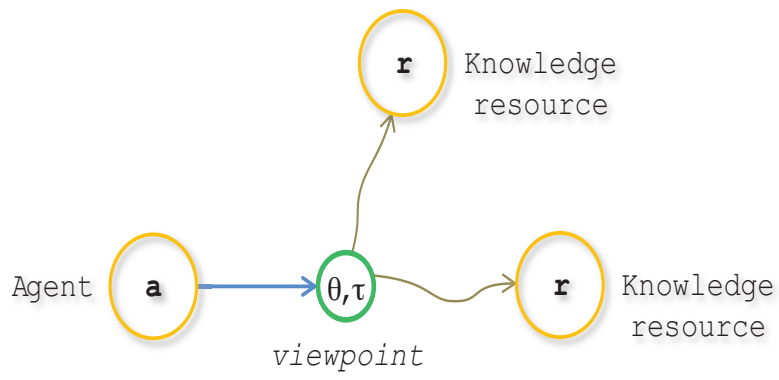

Fig.1: A "viewpoint”. The straight arrow gives the provenance; ' $\theta$ ' gives the semantics e.g., 'authorship', 'matches', 'similar'; ' $\tau$ ' gives the time stamp

\footnotetext{
${ }^{3}$ In this context we prefer « construction and exploitation » to the terms «production and consumption » that are often popular within the description of network protocols.

${ }^{4}$ Notice that a significant part of this intelligence is due to the collective contributions by other Agents. One of the assumptions is that the crowd indeed shows a kind of wisdom.
} 
Each Agent declares, constructs subjective proximities by emitting viewpoints. ${ }^{5}$ Those are globally transformed into topological proximity within a graph - called: Knowledge Graph (KG). This graph KG can in turn be exploited by each Agent, according to his/her own subjectivity, the user's subjectivity, by means of transforming it into another Graph called Knowledge Map (KM) that is the result of a functional transformation called: perspective. The perspective may be considered like a rule-based filter that enters the $\mathrm{KG}$ and produces the $\mathrm{KM}$ on behalf of a single or collective Agent: proximities which are qualitatively declared in the KG by viewpoints are filtered and quantified on demand according to a perspective and transformed into subjective links between knowledge resources - called synapses - that form on turn the KM. This process is depicted in the simplified, yet detailed Fig.2 below.

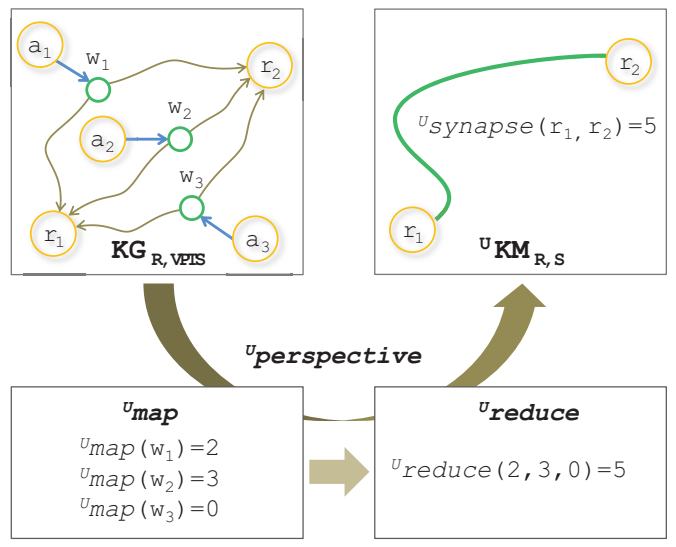

Fig. 2: The process of building a knowledge map ${ }^{U} \mathrm{KM}$, where ' $U$ ' stands for the subjective perspective of the User specifying the ${ }^{\mathrm{U}}$ map and ${ }^{\mathrm{U}}$ reduce functions ${ }^{6}$, i.e. the rules quantifying the viewpoints. In this example, the weights 2,3 and 0 have been respectively assigned ( ${ }^{U}$ map) to the viewpoints $\mathrm{w}_{1} \mathrm{w}_{2}$ and $\mathrm{w}_{3}$, and then these weights have been summed up ( ${ }_{\mathrm{re}}$ duce) in order to produce a synapse weighting 5 between resources $r_{1}$ and $r_{2}$.

\footnotetext{
${ }^{5}$ The viewpoint $\left(a_{1},\left\{r_{2}, r_{3}\right\}, \theta, \tau\right)$ stands for: the agent $a_{1}$ believes at time $\tau$ that $r_{2}$ and $r_{3}$ are related according to the semantics carried by $\theta$. Notice that viewpoint declarations may occur as side effects of other actions, such as clicking an URL. Artificial Agents (e.g.: mining algorithms or theorem provers from the Semantic Web) may also contribute to declare dynamically viewpoints.

${ }^{6}$ Notice that the Map-Reduce labels have been chosen with a view to the dataflow future implementation of the dynamic process. We believe that this implementation will be needed for performance, since the transformation $\mathrm{KG}->\mathrm{KM}$ is in principle performed continuously for each Agent and dataflow processes are naturally concurrent. However, the conception of processes in terms of dataflow with the three classical operators (maps, accumulators $=$ reducers, filters) is also an alternative to the classical object-oriented one. Dataflow concurrency may make explicit other interesting behaviors (and their corresponding interpretations) that may be more similar to the ones of the brain.
} 
The following concrete points should be noted:

1. ' $\theta$ ' takes currently values within a non-ambiguous and limited set (e.g.: 'matches', 'authorship' ,'similar') ${ }^{7}$,

2. default functions for quantifying and aggregating the viewpoints according to ' $\theta$ ' and other parameters have been defined, so that only expert users will possibly feel the need to tune them according to their needs ;

3. there is in principle no boundary in the choice of functions and rules for weighting and aggregating viewpoints in order to produce a perspective: we kept the current version simple, but more complex filtering processes may be conceived.

A perspective corresponds not only to the "once for all" user's profile; rather to a dynamic focusing lens that users exploit to interpret the Knowledge Graph evolving with the time.

So, the subjectivity impacts the KM - that is the Information structure where Agents navigate - in two phases: when constructing the KG (Agents declare autonomously subjective proximities) and when transforming it into the KM (Agents may define their own perspective for transforming $\mathrm{KG}$ into $\mathrm{KM}$ ).

We adopt the vision of the authors of ViewpointS, that the interactive processes intertwining the construction and exploitation of the Knowledge Graph - Knowledge Map may possibly become a particularly effective source of informal learning, in particular serendipitous learning by using a perspective that enhances the proximity of elements in the KM that are relevant to the Agent.

We add to their vision the conjecture that memorizing and exploiting -by means of the perspective- the mental states of the Agents producing the $\mathrm{KG}$ as well as the ones consuming the KM may offer interesting enhancements in terms of serendipitous learning.

This assumption is supported by a previous theoretical study [6] where the ViewpointS authors have explored in a mock-up, simulated situation - called "the three princes of Serendip" - how they can graphically assess abstract concepts (i.e. colors) and promote serendipitous learning of these concepts. It was interesting to notice that such learning occurs as a consequence of free navigation in maps that have been influenced by other Agents on the basis of other Agent's competence, conviction, knowledge, without any explicit intention to teach. Once more: the external context influences learning even without any internal decision or intention.

\section{Web Science: studying societies of Humans by tracing their behavior on the Web}

A few years ago, a movement was launched about the notion of Web Science [16]. The main objective was to give a status of Science to the studies interested in understanding and predicting human individual and collective behavior, by means of dis-

\footnotetext{
${ }^{7}$ Practically: users may tune a limited number of sliders in order to define the filter concerning $\theta$ for building their own perspective.
} 
tinguishing those initiatives from the very successful recent technical advances qualifying as Web Technologies.

The difference is certainly not a minor one: a science profits but does not coincide with its related techniques or technologies. In particular, a natural science looks into natural phenomena in order to understand and predict them as much as possible by iterating the cycle of conjectures and refutations [17]. One of the pillars of the process consists in the performance of Bayesian significance tests. However, before the formulation of a conjecture, one should have a descriptive idea about the phenomena at stake and the variables that may represent them in a significant way. This pre-study usually precedes inferential statistics.

The question then arises naturally: is the Web a "natural phenomenon"? Insofar the Web consists "only" of computers and telecommunication links, it is more a complex artifact as a natural phenomenon. However, IF the Web - as we assume to be the case - consists of a few billions of networked computers AND humans THEN it may be studied as a natural, social, complex phenomenon.

The metaphor of autonomous Agents helps: these entities may abstract autonomous behavior in societies of communicating humans and machines. These evolving behaviors may be studied in order to understand and forecast interesting individual and collective phenomena ${ }^{8}$.

We assume that individual and collective knowledge construction and exploitation such as those occurring on the Web are interesting social processes, contributing significantly to our understanding of intelligence. We are also convinced that intelligence is not "all or nothing" but may only be evaluated differentially. Finally, we are convinced of the important influence of mental states and personality traits ${ }^{9}$ in human individual and collective intelligence.

Activities on the Web are traceable as it is the case of search in Google, interactions in social media but also construction and search within the ViewpointS environment. Inferences about proximity made by ViewpointS are possible; a personalized Knowledge Map (KM) may be measured against one more generic.

Therefore, we conjecture that ViewpointS-based interactions suitably traced, studied and exploited may usefully contribute to the progress of Web Science, in the above outlined sense of the science of complex sociotechnical systems including humans.

\footnotetext{
${ }^{8}$ Notice that in distributed systems with no centralized control the closed world assumption does not hold for each Agent so that even a set of distributed communicating artificial Agents may be considered a collection of autonomous entities that present emergent phenomena similar to the natural ones.

9 Personality traits are long-term properties of humans. Mental states are on the contrary ephemeral, time-related. In this paper we only consider mental states as consequences of emotions, motivation, commitment, sentiment.
} 


\section{The availability of new ways to measure brain mental states and their relations to learning}

The concepts developed by Claude Frasson and his group at the University of Montreal about "measurable mental states" indeed pave new ways to assess brain behavior during human-computer interactions, in particular during (formal) learning sessions. Many preliminary results are available (for instance: [18]) confirming that physiological measures may be taken with relative ease. These enable to understand and forecast emotions and mental states so that a suitable feedback process may modify the foreseen plan of the learning session in order to better fit the learner's mental state. However, as much as we are aware, no previous investigation was done on learning as a side effect of free interactions, i.e.: informal and serendipitous learning.

In free interactions, there is no "plan of the learning session" as there is no explicit intention to learn or to teach when exploring the information space available. Therefore, if measurable mental states are made available, the only possible effect they may have on the learner's behavior is in modifying the Information space, in our context: $K M$. In the following, we outline shortly how the interplay between KG and KM may enhance the availability of particular elements in the KM as they are considered more "proximal to the user in his/her particular mental state" thus eligible for navigation and the consequent learning effects.

\section{Exploiting the mental states of the internauts when analyzing the serendipitous knowledge acquisition processes}

In the ViewpointS approach, exploiting the Knowledge Map issued from the viewpoints stored in the KG is done by choosing perspectives, i.e. valuation rules for these viewpoints according to their types, dates, provenance etc. Each perspective may fit the current needs or curiosities of a user (who can be viewed as "perspective rider") and leads to a specific KM (Knowledge Map).

The construction of the KG as well as the KM are dynamic: interactions of other Agents may update the KG and each user Agent may regularly update his/her KM by applying a filtering process adopting a perspective.

For instance, one may filter viewpoints in the KG by provenance: give more weight to the ones attributed to notorious personalities (high reputation), or either attribute a weight inversely proportional to the emission time, introducing a kind of forgetting.

Assuming that both criteria in the example have been applied in a perspective, synapses in the KM will possibly be reinforced by the most recent viewpoints declared by the personalities with high reputation.

Hereafter some very preliminary description of how to link viewpoints to Serendipitous Learning enhancement:

Let us suppose we have means for characterizing the mental state of the Agents at any time they interact with the KG (construction) or with the KM (exploitation). 
If we assess and store the mental state of the viewpoints emitters by means of typ-

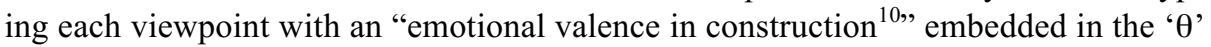
parameter outlined in Fig. 1: we can exhibit, through the mechanism of perspective, which knowledge paths (i.e.: paths in the KM, synapses) emerge when a specific emotional valence is selected within the producers (of knowledge).

This representation of the knowledge produced by a single Agent or a society of Agents not only may reflect the beliefs of these Agents about the world's elements proximities, but also their emotions (or mental states) at the moment they have produced the beliefs. The advantage with respect to traditional profiling or modeling the user's state is that these representations do not necessarily require any particular action by the Agents or by the designer of the experiment, rather they are a simple side effect of interacting with the environment.

Reversely, if we assess and store the mental state of the users by means of typing each perspective with an "emotional valence in exploitation": we can exhibit which knowledge paths = synapses have been followed when a specific emotional valence is selected within the users. Typing perspectives with an emotional valence implies that perspectives become first class resources. This information (perspectives defined by a user when exploiting the KG) - together with the viewpoints emitted by the user within $\mathrm{KG}$ - may represent a powerful, dynamic model of the user's state.

The description of the evolution of KG and KM along the time, for single Agents as well as communities of Agents, may be a rich source of inspiration in order to understand the cognitive state of humans, combining rational - the resources concretely visited - and emotional aspects (mental states).

\section{Examples of analysis of learning by observing human-web interactions}

Serendipitous is an adjective that usually is associated to learning. Learning is usually linked to a "positive" event: changing the person's state from a state of ignorance to a state of competence with respect to a concept or a skill.

In our vision, we postulate that learning may be also associated to the events occurring in the framework of other processes modifying significantly human Agents, not necessarily in a positive way.

We postulate that all the times the "state" of a person is substantially modified by interactions with the environment and there is no explicit intentions by the person, the event may be qualified as serendipitous learning, by associating to the word learning the neutral connotation of "substantial modification of the state of the person", in particular the cognitive or the mental state of the person.

We propose therefore the conjecture that serendipitous learning is crucial in many situations essentially because it represent a very wide class of non-voluntary modifications of the person's state induced by interactions. Three examples - among many

\footnotetext{
${ }^{10}$ As it was noticed before, we are not yet able to specify exactly what we mean by emotional valence: it denotes some classification of a mental state.
} 
possible ones - that we consider not only important but also adequate for investigation consist of:

1. a positive one: science discovery ${ }^{11}$ (learning-discovering a new concept without an explicit intention);

2. a neutral one: political adhesion (learning-acquiring a significant political conviction);

3. a negative one: radicalism (learning-acquiring a new positive attitude-emotion toward facts that are associated to crime in general, for instance: mafia or islamistic criminal actions).

For each of these situations, we envision to perform free navigation experiments within the ViewpointS environment augmented by traces of the mental state of Agents, during both the phases of construction of the Knowledge Graph and of exploitation of the Knowledge Map. Since by definition serendipitous learning is not dependent on a purpose neither by the learner nor by the learning environment, the only way to measure it will be to analyze the KM before and after navigation. Resources (Agents, Documents, Concepts) available to the user have a certain distance with the user-Agent considered as a resource. The distance may change after an interaction, so we may use the distance of a user-Agent to one or more resources as a measure of proximity that evolves with time according to the Agent's rational and emotional state.

Let us consider the case n. 2, perhaps the easiest one: political adhesion. Part of the learners will be changing their sentiment and adhere to a candidate or a party (distance with the candidate or the party's key concepts will be reduced), or vice-versa will change candidate or move to a state of non adhesion as a result of the monitored navigation (distance with the initial candidate or party will increase).

\section{Conclusion}

In this position paper we speculate about the opportunity offered by current physiological measures of emotions and mental states to enrich significantly the construction and exploitation of human cognitive models by adding the emotional dimension. Both construction and exploitation are described within a new context of Agent interaction called ViewpointS, where a single primitive: subjective proximity seems sufficient to unify the Semantic and the Social Web. The major advantage of such an enrichment consists of the fact that the construction and exploitation of brainenhanced models should come at no specific modeling costs except the necessary apparatus for acquiring the physiological signals and is used within free navigation where serendipitous learning seems to be a well established phenomenon.

\footnotetext{
${ }^{11} \mathrm{https} / / /$ en.wikipedia.org/wiki/Role_of_chance_in_scientific_discoveries
} 


\section{References}

1. Carbonnel, J.R.: AI in CAI: An Artificial-Intelligence Approach to Computer-Assisted Instruction. IEEE Transactions on Man Machine Systems 11(4), 190 - 202 (1970)

2. Self, J.A.: Student models in computer-aided instruction. International Journal of ManMachine Studies, 6 (2), 261-276 (1974)

3. Self, J.A.; Bypassing the Intractable Problem of Student Modelling. In Frasson, C., Gauthier, G. (eds.), Intelligent Tutoring Systems: at the Crossroads of Artificial Intelligence and Education, Norwood (N.J.): Ablex, pp. 107-123 (1990)

4. Sefton-Green, J.; Literature Review in Informal Learning with Technology Outside School. NESTA FUTURELAB, report 7, https://www.nfer.ac.uk/publications/FUTL72 last accessed 2017/04/15

5. Lemoisson, P., Surroca, G., Jonquet, C. and Cerri, S.A. ViewpointS: When Social Ranking Meets the Semantic Web. In: Rus, V. and Markov, Z. (eds) FLAIRS 2017 The 30th International FLAIRS Conference, AAAI Press, Marco Island, Florida, USA (2017)

6. Surroca, G., Lemoisson, P., Jonquet, C. and Cerri, S.A. Preference Dissemination by Sharing Viewpoints : Simulating Serendipity. In: Fred, A., Aveiro, D., Dietz, J., Filipe, J., Liu, K. (Eds.) Proceedings of the 7th International Joint Conference on Knowledge Discovery, Knowledge Engineering and Knowledge Management, Volume 2: KEOD, IC3K (2) pp. 402-409 Lisbon, Portugal. (2015)

7. Corneli, J., Pease, A., Colton, S., Jordanous, A., Guckelsberger, C . Modelling serendipity in a computational context. CoRR abs/1411.0440 (2014), https://arxiv.org/abs/1411.0440 last accessed 2017/04/15

8. Van Andel, P.: Anatomy of the Unsought Finding. Serendipity: Origin, History, Domains, Traditions, Appearances, Patterns and Programmability. The British Journal for the Philosophy of Science 45, 2, 631-648 (1994)

9. Rorschach tests, https://en.wikipedia.org/wiki/Rorschach_test, last accessed 2017/04/15

10. Castrogiovanni P., Maffei G., Pasquinucci P.J., Lijtmaer N., Torrigiani G., Cerri S.A., Zampolli A. : Analisi linguistica delle risposte al test di Rorschach di schizofrenici e neurotici e dei rispettivi familiari. I. - Metodologia e primi risultati di un'analisi condotta mediante elaboratori elettronici. Neopsichiatria 34(4), Arti Grafiche Pacini Mariotti, Pisa, Italy, 810-837 (1968)

11. Personality Traits, https://en.wikipedia.org/wiki/Big_Five_personality_traits, last accessed 2017/04/15

12. Nunes, M.A.S.N., Cerri,S.A., Blanc,N. : Improving Recommendations by Using Personality Traits in User Profiles. International Conferences on Knowledge Management and New Media Technology, Graz, Austria, 92-100, (2008)

13. Principles of grouping or Gestalt laws of grouping, https://en.wikipedia.org/wiki/Principles_of_grouping, last accessed 2017/04/15

14. Edelman, G. Neural Darwinism: The theory of neuronal group selection. Basic Books, New York (1987)

15. VygotskyZPD, https://en.wikipedia.org/wiki/Zone_of_proximal_development last accessed 2017/04/15

16. Web Science, http://www.webscience.org/manifesto/ last accessed 2017/04/15

17. Popper, K .; Conjectures and Refutations. The Growth of Scientific Knowledge. Basic Books, New York (1962)

18. Chaouachi, M., Jraidi, I., Frasson, C.: Adapting to Learners' Mental States Using a Physiological Computing Approach. FLAIRS 2015, The 28th International FLAIRS Conference, AAAI Press, Hollywood, Florida, USA (2015) 